\title{
INFLUENCE OF ACCUMULATION LAYER ON RESONANT TUNNELING IN DOUBLE-BARRIER STRUCTURES
}

\author{
E. KACZMAREK \\ Institute of Physics, Polish Academy of Sciences \\ Al. Lotników 32/46, 02-668 Warszawa, Poland
}

\begin{abstract}
The existing theory of the resonant tunneling phenomena in double-barrier structures takes into account the energy quantization in the well confined between the barriers only. In real tunneling structures there is another well, i.e., the accumulation well in the spacer region separating the highly doped region and the double-barrier structure. In the present paper the transmission coefficient for double-barrier structures with an accumulation layer as a function of applied voltage has been derived. The experimentally observed oscillations of the tunneling current can be explained by the obtained quantization of the energy spectrum in the accumulation well.
\end{abstract}

PACS numbers: $73.40 . \mathrm{Gk}$

Recent advances in the technology of growth of semiconductor heterostructures have stimulated numerous studies, both experimental and theoretical, of the resonant tunneling through double-barrier structures $(\mathrm{DBS})$ [1]. Let us recall that the resonant tunneling occurs when the energy of the tunneling electron matches the resonant energy level $E_{\mathrm{R}}$ in the well confined between the DBS barriers. The typical tunneling structure consists of the emitter region, the double-barrier structure and the collector region. In real tunneling structures the emitter region consists of the $n^{+}$type GaAs layer and the slightly doped or undoped GaAs layer, called the spacer. It means that there is an additional barrier formed on the $n^{+-} n$ junction in the emitter region. The height $V_{\mathrm{s}}$ of this barrier is equal to the difference between the Fermi levels in the $n^{+}$type and the $n$ type layers. Usually the spacer is nondegenerate semiconductor and the $V_{\mathrm{s}}$ is comparable with the resonance energy $E_{\mathrm{R}}$. The effective resonance energy, $E_{\mathrm{R}}^{\prime}=E_{\mathrm{R}}+V_{\mathrm{s}}$, becomes larger and the bias required to align the $E_{\mathrm{R}}^{\prime}$ and the Fermi energy increases. Thus, the large accumulation well is formed in the spacer region and one can expect that the energy quantization in the accumulation well may play a significant role.

To draw out the main features of the resonant tunneling through double-barrier structure with accumulation layer we have considered the one-dimensional potential profile created by the boltom of conduction band of the structure under applied voltage (Fig. 1). First, let us take under consideration the essential 


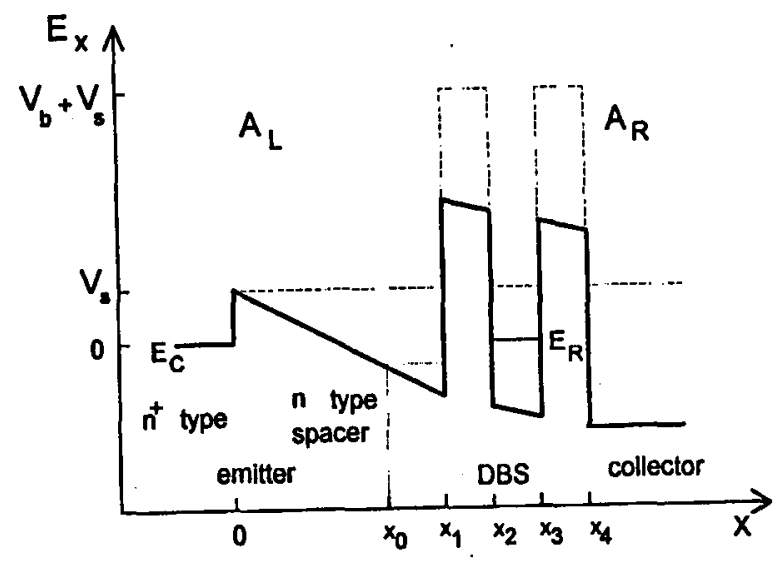

Fig. 1. Profile of the potential energy of an electron in the DBS with the spacer layer without (dashed) and with an applied bias (solid line). Here $x_{0}$ denotes the turning point, $x_{1}=s, x_{2}=s+b_{1}, x_{3}=s+b_{1}+w, x_{4}=s+b_{1}+b_{2}+w$.

difference between the boundary conditions for the electron wave function with the energy above $(E>0)$ and below $(E<0)$ the bottom of the $n^{+}$type layer conduction band. In the latter case the electron cannot penetrate the region on the left hand side of the turning point $x_{0}$ and its state in the accumulation well is described by the Airy function $\mathrm{Ai}(x)$ vanishing exponentially for $x<x_{0}$. The current density for such state is equal to zero. It vanishes also in the collector region due to the continuity of the current density. Thus, the tunneling can occur only for energies above the conduction band bottom of the $n^{+}$type layer. For $E>0$ the transmission coefficient can be calculated by the standard procedure [2]. To this end we have taken the electron wave function as linear combinations of the plane waves in the $n^{+}$type and the collector regions and as linear combinations of the Airy functions $\mathrm{Ai}(-z)$ and $\mathrm{Bi}(-z)$ in the spacer and DBS regions. Here,

$$
z=\alpha\left[x+\left(E-V_{\mathrm{i}}\right) / e F\right]
$$

where $\alpha=\left(2 m^{*} e F / \hbar^{2}\right)^{1 / 3}$ and $V_{\mathrm{i}}=V_{\mathrm{s}}$ both, in the spacer region and the DBS well or $V_{\mathrm{i}}=V_{\mathrm{b}}+V_{\mathrm{s}}$ in the DBS barriers. We have calculated the transmission coefficient $T=\left|A_{\mathrm{R}} / A_{\mathrm{L}}\right|^{2}$, i.e., the ratio of the transmitted wave amplitude $A_{\mathrm{R}}$ to the incident wave amplitude $A_{\mathrm{L}}$. This was done by matching the wave functions and their derivatives in the points of discontinuity of the potential. Using the asymptotic expansions of the Airy functions for large $|z|[3]$ for the states in the DBS region, we have got an expression for the global transmission coefficient $T_{\mathrm{G}}$ in the form very similar to that for DBS without accumulation layer

$$
T_{\mathrm{G}}=\frac{4 \sin 2 \beta_{1} \sin 2 \beta_{2} \sin 2 \beta_{3} \sin 2 \beta_{4}}{D},
$$

where the denominator $D$ is given by 


$$
\begin{aligned}
D= & Q_{1}\left[\frac{1}{T_{1} T_{2}} S_{1}^{2}+\frac{T_{2}}{T_{1}} S_{3}^{2}-2 \frac{S_{1} S_{3}}{T_{1}} \cos 2 \beta_{4}\right] \\
& +Q_{2}\left[\frac{T_{1}}{T_{2}} S_{2}^{2}+T_{1} T_{2} S_{4}^{2}-2 T_{1} S_{2} S_{4} \cos 2 \beta_{4}\right] \\
& +2 Q_{3}\left[\frac{1}{T_{2}} S_{1} S_{2}+T_{2} S_{3} S_{4}-\left(S_{1} S_{4}+S_{2} S_{3}\right) \cos 2 \beta_{4}\right] \\
& +\left(S_{2} S_{3}-S_{1} S_{4}\right) \sin 2 \beta_{1} \sin 2 \beta_{4} .
\end{aligned}
$$

Here $T_{1}$ and $T_{2}$ are the transmission coefficients for the single DBS barriers and $S_{i}$ and $\beta_{i}$ are the phase factors. The explicit definitions of the $T_{i}, S_{i}$ and $\beta_{i}$ are given in our previous paper [4].

The whole information on the influence of the accumulation well on the resonant tunneling is enclosed in the functions $Q$ :

$$
\begin{aligned}
Q_{r}= & \pi\left\{\left(\alpha / k_{\mathrm{L}}\right)\left[\mathrm{Ai}^{\prime}\left(-z_{0}\right) \sin \phi_{r}+\mathrm{Bi}^{\prime}\left(-z_{0}\right) \cos \phi_{r}\right]^{2}\right. \\
& \left.+\left(k_{\mathrm{L}} / \alpha\right)\left[\mathrm{Ai}\left(-z_{0}\right) \sin \phi_{r}+\mathrm{Bi}\left(-z_{0}\right) \cos \phi_{r}\right]^{2}\right\}, \quad r=1,2, \\
Q_{3}= & \pi\left\{\left[\left(\alpha / k_{\mathrm{L}}\right) \mathrm{Ai}^{\prime}\left(-z_{0}\right) \mathrm{Bi}^{\prime}\left(-z_{0}\right)+\left(k_{\mathrm{L}} / \alpha\right) \mathrm{Ai}\left(-z_{0}\right) \mathrm{Bi}\left(-z_{0}\right)\right] \sin \left(\phi_{1}+\phi_{2}\right)\right. \\
& -\left[\left(\alpha / k_{\mathrm{L}}\right) \mathrm{Ai}^{\prime 2}\left(-z_{0}\right)+\left(k_{\mathrm{L}} / \alpha\right) \mathrm{Ai}^{2}\left(-z_{0}\right)\right] \sin \phi_{1} \sin \phi_{2} \\
& \left.-\left[\left(\alpha / k_{\mathrm{L}}\right) \mathrm{Bi}^{2}\left(-z_{0}\right)+\left(k_{\mathrm{L}} / \alpha\right) \mathrm{Bi}^{2}\left(-z_{0}\right)\right] \cos \phi_{1} \cos \phi_{2}\right\}
\end{aligned}
$$

where

$$
\begin{aligned}
& \phi_{1}=(2 / 3) z_{1}^{3 / 2}+\pi / 4-\beta_{1}, \quad \phi_{2}=\phi_{1}+2 \beta_{1}, \\
& z_{0}=(\alpha / e F)\left(E-V_{\mathrm{s}}\right), \quad z_{1}=(\alpha / e F)\left(E-V_{\mathrm{s}}+e F s\right), \\
& \operatorname{tg} \beta_{1}=\left\{\left[V_{\mathrm{b}}-\left(E-V_{\mathrm{s}}+e F s\right)\right] /\left(E-V_{\mathrm{s}}+e F s\right)\right\}^{1 / 2}, \quad k_{\mathrm{L}}=\left[2 m^{*} E / \hbar^{2}\right]^{1 / 2} .
\end{aligned}
$$

The functions $Q$ are oscillating functions of energy in the whole range of energy under consideration $\left(E<V_{\mathrm{b}}+V_{\mathrm{s}}\right)$, due to oscillatory character of functions $\sin \phi_{r}$ and $\cos \phi_{r}$ appearing in these functions. The amplitudes of the oscillations depend strongly on the energy of tunneling electron $E$ vs. the height $V_{\mathrm{s}}$ of the spacer barrier: for energies $E>V_{\mathrm{s}}$ the amplitudes of the functions $Q$ are of the order of unity whereas for $0<E<V_{\mathrm{s}}$ these amplitudes are several orders of magnitude bigger.

The functions $Q$ have minima for such values of electron energies for which the prefactors of $\mathrm{Bi}\left(-z_{0}\right)$ are equal to zero: $\cos \phi_{1}=0$ or $\cos \phi_{2}=0$. Thus, the conditions for the quasi-localized resonant energy levels $E_{n}^{ \pm}$in the accumulation well are given by the solutions of the transcendental equations differing in sign of the $\beta_{1}(E)$ term

$$
(2 / 3)\left[(\alpha / e F)\left(E-V_{\mathbf{s}}+e F s\right)\right]^{3 / 2}=(n+1 / 4) \pi \pm \beta_{1}(E) .
$$

These are analogous to the well known condition for the resonant energy levels in the DBS well. Still, the energy level scheme for the accumulation well is much more complicated than that for the DBS well. It depends strongly not only on the 


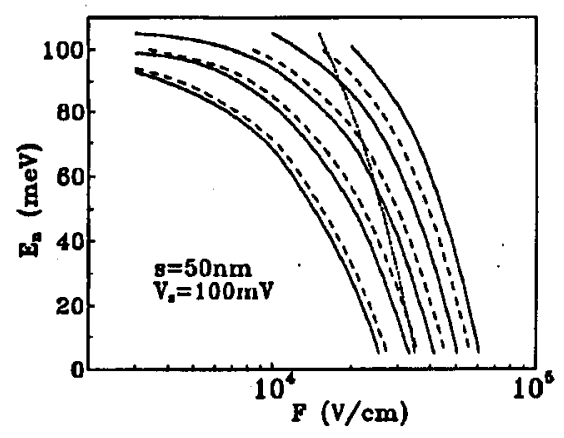

Fig. 2. The energy levels in the accumulation well vs. the electric field $F$ for $s=50 \mathrm{~nm}$, $V_{\mathrm{s}}=0.1 \mathrm{eV}, b_{1}=b_{2}=w=5.6 \mathrm{~nm}, V_{\mathrm{b}}=0.36 \mathrm{eV}$. The solid and dashed lines correspond to $E_{n}^{+}$and $E_{n}^{-}$levels, respectively. The dotted line shows the energy $E_{\mathrm{R}}+V_{\mathrm{s}}-e F s$ for the DBS.

electric field $F$ but also on the DBS and spacer parameters as the latter determine the range of $F$, for which the resonant tunneling occurs. It is very difficult to discuss this energy scheme in general. As an example, the dependence of the energies $E_{n}$ on the electric field for a one set of the parameters is shown in Fig. 2.

Finally, let us discuss briefly how the quantization of energy due to accumulation well influences the resonant tunneling. Let us recall that the transmission coefficient $T_{\mathrm{G}}$ for the DBS alone has the sharp maximum at the resonance energy $E_{\mathrm{R}}$ determined from the equation $S_{1}=0$. IIere the $T_{\mathrm{G}}$ has the maximum when also the functions $Q$ reach their minimal values. In other words, the resonant tunneling occurs when the resonant level $E_{\mathrm{R}}$ in the DBS crosses the resonant levels $E_{n}$ in the accumulation layer (see Fig. 2).

Concluding, the quantization of energy in the accumulation well causes the oscillations in the tunneling current, as observed in [5]. The number and the period of the oscillations depend nearly exclusively on the spacer width. Finally, the proportionality of the global transmission coeflicient to the spacer transmission coefficient should reduce the magnitude of the main current peak.

This work was supported by the State Committee for Scientific Research (Republic of Poland), grant No. 2 P03B 03508.

\section{References}

[1] B. Ricco, M.Ya. Azbel, Phys. Rev. B 29, 1970 (1984).

[2] E.O. Kane, in: Tunneling Phenomena in Solids, Eds. E. Burstein, D. Lundquist, Plenum Press, New York 1969, p. 1.

[3] Handbook of Malhematical Functions, Eds. M. Abramowitz, I.A. Stegun, Dover Publications, Inc., New York.

[4] E. Kaczmarek, W. Szkiełko, Acta Phys. Pol. A 80, 441 (1991).

[5] S.A. Vitusevich, T. Figielski, A. Mąkosa, T. Wosiński, A.E. Belyaev, R.V. Konakova, L.N. Kravchenko, Acta Phys. Pol. A 87, 377 (1995). 